\title{
Review Article \\ Evolution of Satellite Communication Antennas on Mobile Ground Terminals
}

\author{
Haifeng Zhou, Mingchinq Jong, and Guoqiang Lo \\ Institute of Microelectronics, Agency for Science, Technology and Research (A STAR), 11 Science Park Road, \\ Singapore Science Park II, Singapore 117685 \\ Correspondence should be addressed to Haifeng Zhou; hfzhou1984@gmail.com
}

Received 4 June 2015; Accepted 26 July 2015

Academic Editor: Weixiang Jiang

Copyright (C) 2015 Haifeng Zhou et al. This is an open access article distributed under the Creative Commons Attribution License, which permits unrestricted use, distribution, and reproduction in any medium, provided the original work is properly cited.

\begin{abstract}
Getting connected whenever and wherever you travel is not kind of luxury any more. Satellite communication researchers are making it a reality to bring you data, video, and voice services when you are away from home, out of office, or on a long journey. Satellite communication antenna mounted on mobile ground terminals is an essential prerequisite of successful connectivity. In this paper, we review the evolution of this kind of antenna in its historical context and outline the major research achievements on ground mobile terminals. Many striking demonstrations and prototypes are revisited to understand the emerging technologies and assess their potential towards practical implementations. The future trends and challenges are also discussed.
\end{abstract}

\section{Introduction}

Owing to promise of global coverage and excellent versatility, satellite communications (SatCom) constantly tailor the basic telecommunication needs of many countries even though humankind had entered into the era of broadband internet and optical fiber communication. $60 \%$ of the nearly 3,600 operating satellites as of May in 2015 are communications satellites. The TAURI group of satellite industry association (SIA) revealed that the satellite consumer equipment including mobile satellite terminals grew $22 \%$ in 2014 and mobile satellite services (voice and data) 6\% [1]. This report manifests that mobile satellite communication (MOST) becomes a key growth point of the overall satellite industry which only had a growth of $3 \%$ in the same year $[1,2]$. As illustrated in Figure 1, satellites are devoted to constantly and efficiently connect people in airplanes, ships, terrestrial vehicles, and so forth [3,4]. Passengers holding smart phone or lap-top in the air would benefit in-flight connectivity to access internet or entertain streaming video. Travelers on fast moving vehicles demand constant connectivity to keep in touch and make important calls [5, 6]. Ship personnel may benefit from crew connectivity with the latest maritime information such as chart updates, engine monitoring, and weather routing broadcast. The backpack terminals have to be quickly and reliably deployed to support connectivity in harsh and emergent conditions. To fulfill these missions, the solutions leveraging on terrestrial mobile communication infrastructures, typically like long-term evolution (LTE), are relatively slow, expensive, and regional and are not easily scalable. SatCom is an attractive alternative because of its superior reliability, cost effectiveness, excellent accessibility, and scalability [7].

Without any exception, SatCom is always delivered by antennas that transmit and receive the modulated radiofrequency (RF) carriers through absorptive atmosphere [8]. Apparently, the uplink and downlink antenna systems are the key enabling payload on satellite terminals [9]. There is little work in literatures to systematically review MOST antennas and update new knowledge added to this field. On the other hand, MOST antenna is a flourishing research area that attracts continuous market interest. This paper focuses on the SatCom antennas on mobile ground terminals, scrutinizes the matured product in the market, retrospects the evolution of fundamental design concepts, evaluates the applicability of the emerging technologies, and discusses the future trends and challenges.

First of all, the antenna electrical performance must comply with the basic mission requirements of SatCom $[8$, 10, 11]. (a) SatCom is kind of long-distance point-to-point 


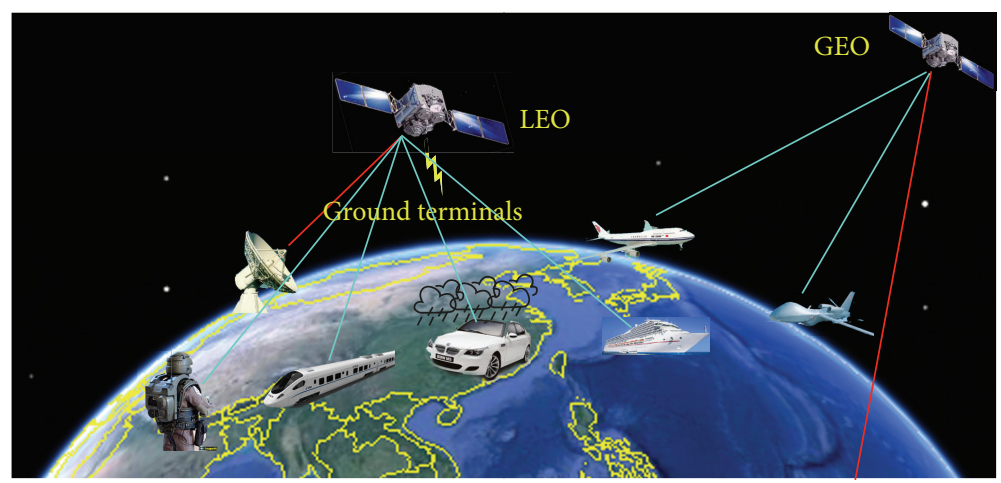

FIGURE 1: Various configurations and services of modern mobile satellite communication.

radio link with a line-of-sight propagation mode. A SatCom antenna must have good enough beam quality, including high gain, narrow beamwidth, suppressed sidelobe, and low cross-polarization, to highly concentrate on the communication object. With limited electric power supply and given microwave path, a high gain means a high effective isotropic radiated power (EIRP) of transmitting antenna or a high gain/system noise temperature $(G / T)$ of receiving antenna. Narrow beamwidth and suppressed sidelobe are critical to prevent transmitting signals to and receiving them from undesired directions, which may cause multipath fading and unwanted interference to other SatCom systems. (b) SatCom has the absorptive atmosphere as its transmission medium, which imposes constraint on frequency usage [12]. In addition, frequency allocation at different regions and services are managed by international telecommunication union (ITU) because of such a limited natural resource. Currently the $K_{u}$ band dominates in the civilian applications. More than 240 satellites in the sky were equipped with $K_{u}$ band payload. It features in the use of very small aperture terminals, the bandwidth availability, and global coverage. $X$ band is mainly used for military purpose. Notably more and more $K_{a}$ band satellites are deployed worldwide, which potentially promises a larger data rate than the congested $K_{u}$ band [13]. (c) Polarization is another important aspect. Linear polarization (LP) and circular polarization (CP) are commonly used for SatCom. Basically LP transmitter calls for strict polarization alignment on the receiver side. CP signal passes through rain with less loss. The orthogonality of both polarizations allows increasing channel capacity by sharing the same frequency. So far LP dominates in $K_{u}$ receiving terminals, whereas CP becomes popular in $K_{a}$ ones.

Other than these, MOST sets extra demands on antenna performance. (a) The beam scanning capability is foremost critical. The relative motion of position and orientation between SatCom objects demands that the antenna should be swiftly steerable to establish and maintain the link. A typical example is the satellite call. Unrestricted mobility must be provided to get continuous service both in the short term during calls and in the long term across large geographies. (b) In a variety of environments, portability is a limiting factor of effectiveness and practicality. It remains a challenge to put bulky and heavy antennas on small aircrafts such as light business jets. The massive protuberance on the aircraft adversely affects the platform's aerodynamic performance. In both military and disaster management situations, the antenna may be backpacked on the mobile personnel so that lightweight is indispensible for practical usage [14].

Many companies have launched their versions of products. Table 1 presents an incomplete list of those marketavailable ones. The specifications come from the datasheets released by vendors. By and large, this list is used to understand the following features and requirements of a marketable MOST antenna. (a) The conventional dish antenna and the phased array antenna are the dominant technologies in commercially available products. (b) As mentioned above, $K_{u}$ band antennas always work with linear polarization. (c) The dish antennas generally have outstanding electrical performance in G/T and EIRP but with very high profile. (d) An emerging technology is competitive only if it supports full azimuthal angle scanning and sufficiently wide elevation angle. (e) A height of less than $15 \mathrm{~mm}$ may be regarded as a criterion of low profile. Keeping these in mind, we can assess the readiness and competitiveness of an emerging technology.

Let us start to review the evolution of antenna concepts. The working principle and basic structures of each are briefly introduced first. Followed is the typical demonstration or prototyping to understand the status of technology. The advantages and deficiencies are discussed as well. Dish antenna, phased arrayed antenna, and reflectarray antenna are elaborated first as they had experienced long-term development yet. The metamaterials-inspired concepts are reviewed separately since that is where recent innovations are converged. Based on the comprehensive survey, our understanding on the future trends and challenges is presented.

\section{Dish Antenna and Its Derivatives}

The mainstream type of antenna used at ground station and on the geostationary satellites to transmit/receive radio frequency $(\mathrm{RF})$ wave is the so-called dish antenna. A dish mainly adopts a parabola shape to reflect the incoming parallel beams to a focal point on a receiving terminal. The antenna gain $G$ is approximately related to the dish diameter $D$ by $G=\eta(\pi D / \lambda)^{2}$, where $\lambda$ and $\eta$ are the wavelength and antenna efficiency, respectively. $\eta$ accounts 
TABLE 1: The products of mobile antennas for satellite communication on the move in the market.

\begin{tabular}{|c|c|c|c|c|c|c|c|c|}
\hline Product & Technology & Freq. & Polarization & $\begin{array}{c}G / T \\
(\mathrm{~dB} / \mathrm{K})\end{array}$ & $\begin{array}{l}\text { EIRP } \\
(\mathrm{dBW})\end{array}$ & $\begin{array}{c}H \\
(\mathrm{~cm})\end{array}$ & Coverage & $\begin{array}{l}\text { Application or } \\
\text { features }\end{array}$ \\
\hline TracVision A9 & $\begin{array}{c}\text { Hybrid } \\
\text { phased } \\
\text { array }\end{array}$ & - & - & - & - & 12.7 & {$[31,57] / 360$} & $\begin{array}{l}\text { In-car } \\
\text { entertainment }\end{array}$ \\
\hline ViaSat 2540 & Horn array & $K_{a}$ & Dual-linear & 12.5 & 43.5 & 21 & {$[0,75] / 360$} & $\begin{array}{l}\text { In-flight } \\
\text { connectivity }\end{array}$ \\
\hline RaySat SR5000 & - & $K_{u}$ & Linear & 8.0 & 44.7 & 19 & {$[25,80] / 360$} & Small vehicles \\
\hline RaySat ER7000 & - & $K_{u}$ & Linear & 11.8 & 55.0 & 29.8 & {$[0,90] / 360$} & $\begin{array}{c}\text { Trains and large } \\
\text { vehicles }\end{array}$ \\
\hline $\begin{array}{l}\text { RaySat } \\
\mathrm{SR} 300 K_{u}\end{array}$ & $\begin{array}{l}\text { Flat panel } \\
\text { array }\end{array}$ & $K_{u}$ & Linear & 6.0 & 44.7 & 26.2 & {$[0,90] / 360$} & $\begin{array}{c}\text { Small } \\
\text { vehicles/vessels }\end{array}$ \\
\hline $\begin{array}{l}\text { RaySat } \\
\text { SR300K }\end{array}$ & $\begin{array}{l}\text { Flat panel } \\
\text { array }\end{array}$ & $K_{a}$ & Circular & 9.0 & 49.0 & 26.2 & {$[0,90] / 360$} & $\begin{array}{c}\text { Small } \\
\text { vehicles/vessels }\end{array}$ \\
\hline $\operatorname{MOST} K_{a}$ & $\begin{array}{l}\text { Shaped } \\
\text { reflector }\end{array}$ & $K_{a}$ & Circular (fixed) & 14.0 & 51.0 & 28 & {$[6,90] / 360$} & $\begin{array}{c}\text { Broadband GEO } \\
\text { Comm. }\end{array}$ \\
\hline $\operatorname{MOST} K_{u}$ & $\begin{array}{l}\text { Shaped } \\
\text { reflector }\end{array}$ & $K_{u}$ & Linear (adjust) & 11.3 & 48.0 & 28 & {$[6,90] / 360$} & $\begin{array}{c}\text { Broadband GEO } \\
\text { Comm. }\end{array}$ \\
\hline SPITFIRE & - & $K_{u}$ & $\begin{array}{l}\text { Adjustable } \\
\text { linear }\end{array}$ & 10.7 & 44.5 & 20 & {$[0,90] / 360$} & Small business jets \\
\hline SOMA-800D & $\begin{array}{l}\text { Up-looking } \\
\text { dish }\end{array}$ & $K_{u}$ & $\begin{array}{l}\text { Adjustable } \\
\text { linear }\end{array}$ & 17.4 & 54.0 & 58 & {$[10,90] / 360$} & Emergency mobile \\
\hline $\begin{array}{l}\text { ACS-COTM- } \\
300\end{array}$ & Stub array & $K_{u}$ & Dual-linear & $10-13$ & $47-50$ & 11 & {$[20,90] / 360$} & Constant Comm. \\
\hline $\begin{array}{l}\text { TTI SOTM- } \\
\text { KU08A }\end{array}$ & $\begin{array}{l}\text { Passive } \\
\text { radiator }\end{array}$ & $K_{u}$ & Dual-linear & 11 & 42 & 20 & {$[20,70] / 360$} & Vehicular SatCom \\
\hline Thin Sat 300 & - & $K_{u}$ & Dual-linear & $10-13$ & $49-52$ & 11 & {$[20,90] / 360$} & $\begin{array}{l}\text { Connectivity } \\
\text { in-route }\end{array}$ \\
\hline $\begin{array}{l}\text { SWE- } \\
\text { DISH/SAAB }\end{array}$ & $\begin{array}{l}\text { Center-fed } \\
\text { dish }\end{array}$ & $K_{u}$ & Linear & 19 & 54 & 130 & {$[10,120] / 360$} & $\begin{array}{c}\text { Many identified } \\
\text { platforms }\end{array}$ \\
\hline DRS & Dish & $K_{u}$ & Linear & 15 & 50 & 91 & {$[0,90] / 360$} & $\begin{array}{c}\text { Highly dynamic } \\
\text { environment }\end{array}$ \\
\hline MSA-T & $\begin{array}{c}\text { MTM } \\
\text { scatterer }\end{array}$ & $K_{a}$ & Selectable & $8.3-12.7$ & 43.5 & 3 & {$[-65,65] / 360$} & SatCom terminals \\
\hline
\end{tabular}

for all imperfections, including losses, uneven illumination, and spilling off at the dish edges. A moderate $\eta$ can only be achieved by careful manufacturing to control the fluctuation of the parabolic surface well within $\approx \lambda / 50$. In addition, the feed horn must be accurately located at the phase center of the focused beam. These are chief factors that cause dish antenna to be expensive [15]. Parabolic dish antennas are able to provide very high levels of gain and directivity, which makes it almost exclusively used in many applications, such as direct broadcast television, terrestrial microwave links, satellite uplinks, and radio astronomy.

The steerability of dish antenna is commonly acquired by mounting it on a mechanical gimbal. The gimbals are mainly oriented in two orthogonal axes: $X-Y$ or azimuth-elevation. The former does not have the keyhole problem near its zenith position because the fixed axis $X$ points to the horizon but has a large sweep volume for a given antenna aperture. The latter provides a cost-effective and less complicated antenna system for tracking satellite vehicles once the keyhole problem is successfully alleviated [16]. Moreover, it has substantially smaller sweep volume and overall size than a corresponding $X-Y$ gimbal antenna.

Research on mechanical dish antenna for MOST becomes less in that this field is more engineering than science now. However, the effort that people devotes to the miniaturization and modification of mechanical dish antenna does not end [17-21]. For example, the low-profile $K_{a}$ band antenna marketized by the company "mobile satellite communication" provides us with a feel of this direction [21]. As shown in Figure 2, the antenna reflector is structured to a fraction of dish and placed at the center of the dome to maximize the antenna aperture for high gain purpose. It is tiltable to change the elevation angle. The front-end electronics are behind the antenna reflector. The feeding antenna pops out its horn from the bottom of the reflector and illuminates a hyperboloidal secondary reflector at the focus of the reflector. All the components are mounted on a rotatable stage to acquire the scanning capability at azimuthal angles. This configuration is essentially an off-axis Cassegrain antenna [22], in which the feed antenna does not directly illuminate the main dish so 


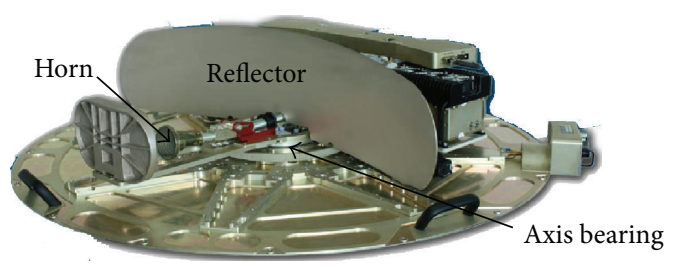

Figure 2: A low-profile design of mechanical dish antenna to fit the limited design space in the dome [21] (used courtesy of MOST Corporation (C2012).

that the main spilling-over sidelobe is filtered before arriving at the main dish. This design features in a small aspect ratio (1/4) by appropriately arranging antenna building blocks.

Gimbal mounted dish antenna is certainly effective in electrical performance. But the bulky and heavy mechanics are not ideal for MOST. The mechanical servos, gears, drive motors, and belts require time-consuming, expensive maintenance, and robustness to conquer erratic movement. For military use, it also has bad invisibility to radar detection. The big head of the "predator" in Figure 1 is a good example, which is specifically designed to accommodate a dish antenna. For airborne terminals and vehicles on rugged terrain, it is almost compulsory to achieve small volume to avoid protrusion outside the aircraft and electronic scanning to assure fast and precise tracking.

The early equation indicates that $G$ is almost just determined by antenna aperture size. Significant volume reduction can only be from the profile thickness, which directly leads to flat-panel design. Flat-panel antennas outperform their larger predecessors through the miniaturization of all possible functional parts in microwave system. All transmission, reception, conversion, cooling, and amplification equipment are embedded within a single unit to achieve low profile [23]. The remaining text will primarily focus on those concepts which promises flat-panel designs.

\section{Phased Array Antenna}

A phased array antenna (PAA) is composed of a number of radiators, a number of phase shifters, and a feeding network. It is well known that the radiation pattern of such a multipleantenna system can be reinforced in favorite directions and suppressed in undesired directions by appropriately feeding the relative phases of the constituent antennas. The frontend electronics include power amplifier on transmitter side and/or low noise amplifier on receiver side. The feeding network takes various forms but basically consists of a combination of serial and parallel connections for power division. As far as a transmitting radiator is concerned, the RF signal is generated by mixing a low intermediate frequency (IF) signal and a local oscillator (LO) signal via upconversion. The phase shifting function may be implemented at different stages, after the LO path, after IF path, or after mixer [24]. Different arrangements have difference in the following aspects: (a) the number of mixers, (b) the number of power combiners/dividers, and (c) the operation frequency that the power combiners/dividers operate. The outcome is different system complexity and power consumption.

The way of using PAA for mobile SatCom purpose is diverse. The extreme case purely uses passive fixed arrayed antenna to achieve high electrical performance and mounts it onto a movable stage for beam scanning purpose [25]. A flat array antenna has a limitation of scanning loss factor, which is inversely proportional to the sine of the scan angle. Keeping the mechanical way to implement beam scanning can avoid this limitation. The antenna volume and scanning speed do not benefit much from antenna array. Another case employs PAA with 1D beam steerability for elevation angle scanning [26]. The azimuth angle scanning is still realized mechanically. For instance, the company MOST replaced the squeezed dish in Figure 2 by slot array antenna but still inherited the mechanical goodies in their latest $K_{a}$ terminals for the enabling technology of high throughput satellite (HTS). This partial use is sufficient to reduce the antenna system height. The ideal case is to implement both elevation and azimuth angles scanning in a fully electronic way. Without the need of any mechanical moving parts, the resulting beam direction can be controlled and directed instantaneously towards any direction.

It should be emphasized that advanced digital processors greatly stimulated the growth of PAA [27-29]. In digital beam forming architectures, the received/transmitted signals down/upconverted from/to each radiator are digitalized at baseband and directly processed in a computational and programmable environment, such as DSP and FPGA. The progressive phase shift between antenna elements is controlled in the digital domain so that analog phase shifting is unnecessary. Because the beam forming is performed within the digital unit, various signal processing operations are available. Hence, digital beam forming is regarded as the most flexible and versatile scheme for PAA, which can accomplish sidelobe minimization, interference cancelation, and multibeam formation without changing any physical architecture.

PAA suitable for SatCom is due to its potential flatness. The modules are usually fabricated on multilayered printed circuit boards (PCBs) [30, 31]. Adopting conformal design for arrayed antenna also allows being hosted on the fuselage of an aircraft or the superstructure of yacht $[32,33]$. Another feature is the scalability because of its modular nature [34]. Once the first module prototype is demonstrated, more modules can be added within minimum down time. This is important to reduce the development cost and extend the application scenarios. The disadvantage of PAA, however, is their large hardware footprint and high cost for the dedicated transceiver module. The phase shifters have to be properly designed to prohibit the beam squinting in which the beam direction may considerably differ at receiving and transmitting frequencies. PAAs also diminish in efficiency at millimeter-wave frequencies due to the use of transmissionline feeding networks which become increasingly lossy at high frequencies [35]. All in all, the key challenge has been to miniaturise all the components involved and improve the performance while reducing the manufacturing cost to an economical level. 


\section{Reflectarray}

It has been long time since reflectarray became a category of antenna to planarize parabolic dish antennas $[36,37]$. However, flat reflectarray that leverages on printed circuit technology is fairly new. The most critical segment in a microstrip reflectarray is an ultrathin, flat reflecting surface, which is composed of many adjustable microstrip structures (typically as textured metallic surface) and illuminated by a feeding source suspended above. In order for high gain toward a special direction, the phase delay over the path from the illuminating feed is compensated by the tunable microstrip patches to satisfy the condition for constructive interference in a targeted radiation angle. The microwave shines on the reflecting surface, experiences phase compensation, and reradiates the energy into space. The use of reflectarray for SatCom application had been vividly depicted in Figure 3 in the work scope of the RETINA (reliable, tunable, inexpensive antenna by collective fabrication processes) project supported by the European Commission [38]. The team aims at producing a reliable and low-cost SatCom system for aircraft. The system is designed to provide in-flight internet services related to passenger web connectivity and safety. The placement of the reflectarray is very impressive because very limited bulky part protrudes from the aircraft fuselage to affect the aerodynamic performance.

The capability of individual elements in the reflectarray is of great importance to compensate for the phase differences of the incoming spherical wave from the feed. The phase shift can be achieved by either resonant or nonresonant structures [39]. The resonant way usually uses tunable resonators with simple metallic patches loaded, where the phase changes rapidly around the resonant frequency due to the change of effective electrical length. Because of the high frequency dependence of resonating effect, this method has limited bandwidth. The nonresonant way is equivalent to using a section of tunable transmission line, where the phase shift changes by varying the transmission line length. An equivalent transmission line perpendicular to the reflection surface is usually implemented with multiple stacked layers, which are lossy and complicated in geometry and are not friendly to accommodate tunable elements. Hence, the resonant way is more popular in demonstrations.

At a given frequency, the phase change should be tunable over an entire phase period of $360^{\circ}$ to sufficiently compensate any possible phase delay. On the other hand, a slower slope of phase change versus frequency is also desired, which can benefit device manufacturing and operation bandwidth. Early resonator-based designs are mostly limited to $180^{\circ}$. It was found that a single-layer structure with multiple resonance elements is able to offer a linear and slowly varying phase change over a broad phase range, which covers full $360^{\circ}$ $[40,41]$.

The resonator-based reflectarray is very much similar to the frequency selective surface (FSS) which alters its electromagnetic properties from an electrical conductor to an artificial magnetic surface. The main difference should be in physical dimension. The latter actually falls into the metamaterials category that we will mention later. We believe that using FSS as reflection surface is helpful to suppress surface wave and improve the radiation beam quality in sidelobe. This direction deserves deep exploration.

Compared to PAA, the spatial feeding method eliminates the energy loss and design complexity of a feeding network. The phase adjustment capability of microstrip allows the reflective surface to maintain flat or conformal to its mounting structure without consuming a significant amount of volume and mass. The low bandwidth performance is the main factor that limits the usage of reflectarray [42]. The bandwidth is affected mainly by the narrow-bandwidth patch elements, the phase errors related to the patch size change, and also by the feed antenna bandwidth, the array element spacing, and dielectric materials of the reflectarray substrate [43]. In addition, reflectarray antenna may also have a null radiation angle towards the feeding horn. It remains a challenge to broaden the scanning range of elevation angles.

\section{Metamaterials Antenna}

Metamaterial (MTM), as an emerging technology, has been extensively investigated in the past decade. MTMs exhibit unique macroscopic properties that natural materials do not have, for example, material with simultaneous negative permittivity and permeability [44]. In geometry, they are usually in the form of engineered structures. For example, LHMs were experimentally demonstrated by incorporating split-ring resonators (SRRs) and metallic thin-wires [45]. Just like the role of atomic structure to macroscopic properties of a material, the structure of unit cell is decisive to the homogenous properties of a metamaterial. If certain tunable element (e.g., varactor, diode) is embedded into the unit cell, the structural or electrical parameters change will be reflected by the macroscopic properties of the MTM, such as effective permittivity/permeability and amplitude/phase retardation per unit length. Consequently, the advent of MTM enlightened RF designer about the miniaturization and functionalization of antenna.

The main difference of MTM from PAA is that the former physically reduces the size and spacing of the constituent units to $<\lambda / 4$. As a result, MTM behaves like a material having homogenous properties, which reacts to microwave through the internal change of the units. The homogenous properties of MTM can be varied by slightly modifying the constituent units that is so-called "meta-atoms," which potentially consumes much less power than phase shifts that perform similar function. By large, the ways of inclusion of MTM fall into the following categories.

5.1. Gradient Index Scheme. Gradient index materials (GRIN), functional materials with gradient spatial distribution of refractive index, have been widely used in imaging optics to control and manipulate light. Recently the concept of GRIN materials has been extended to microwave frequency range at which matured technologies are available to change the MTM atoms enabling this. The GRIN MTM is actually a branch of transformal electromagnetics [46], which is intended to change the ray trajectories in various special 


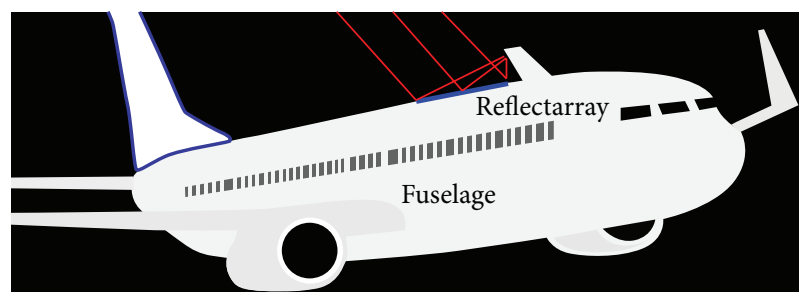

(a)

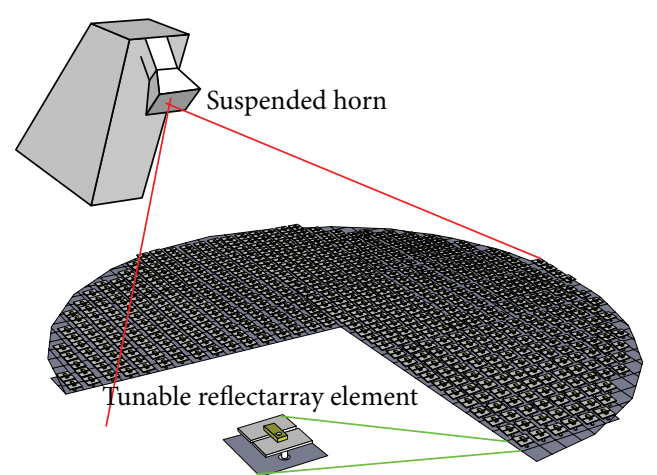

(b)

FIgURE 3: Arrangement of the RETINA SatCom antenna on top of an aircraft fuselage and the basic antenna architecture [38].

ways, such as extreme MTM lens [47] and artificial black holes [48]. However, of SatCom's interest is planar GRIN MTM slab lens, preferably manufacturable by multilayered PCB technologies. The ultimate goal is to collimate waves from a feed source into a pencil beam.

A GRIN MTM slab that monotonically changes phase front can be formed by progressively varying the geometry of successive MTM metallic inclusions. According to geometrical optics, a plane wave passing through a planar GRIN slab acquires different phase retardation normal to slab plane. The beam deflection angle $\theta$ is related to the gradient index $\partial n / \partial x$ by $\theta \approx \arcsin t(\partial n / \partial x)$, where $t$ is the GRIN slab thickness [49]. Figure 4(a) shows an exemplified GRIN MTM slab in $[50,51]$ where a capacitive and an inductive grid are separated by a core layer. With the aid of CST microwave studio, the transmission of Floquet mode through a unit cell can be calculated by imposing periodic boundary conditions. The amplitude and phase of the $S$ parameters are shown in Figures 4(b) and 4(c), respectively. The maxima of the $S_{21}$ parameter in Figure 4(b) indicate the resonant effect of the fundamental Floquet mode while passing through the MTM unit cell. In the vicinity of the resonant frequency, the transmission phase changes rapidly with the structural parameters or electrically tunable elements. Figure 4(c) shows that the transmission phase just has half of the changing range of the reflection phase. As known from the last section, the reflection phase change is a problem yet. This problem for transmission phase change becomes more severe. This is the main constrain of using GRIN slab for beam scanning. In principle, the transmission phase change range can be enhanced by stacking multilayers [52]. However, additional layers may cause significant microwave attenuation. There is little work to address this issue so far.

In experiment, the transmission and reflection properties of a GRIN MTM slab are usually verified by a horn antenna [53]. Apart from a large phase range, a low reflection which causes permanent energy loss is also necessary to improve the antenna efficiency. The impedance matching at the interfaces of GRIN slab is very critical to the beam forming quality [54]. In practice, a low-profile flat-panel design prefers to use microstrip antenna as the radiator instead. The realistic cases mostly use GRIN MTM slab as a partial reflection surface to form a low-profile cavity where a microstrip patch antenna as feeding source is placed between two reflecting surfaces $[50,51]$. The total reflection surface forming the cavity can be either the ground plane of the microstrip antenna or a high impedance electromagnetic surface. Figure 5(a) shows an example of such cavity $[50,55]$ where varactors are placed across the gaps to vary the capacitance. Figure 5(b) shows varactor biasing networks where the partial reflection surface is divided into many regions that can be independently biased. The gain patterns in Figure 5(c) clearly show that beam steering is achieved by raising the biasing voltage, although the steering angle is small.

Because the unit cell size is finite and not infinitely small, the electrical length of the beam shining on the GRIN MTM slab has to be at the scale of several wavelengths. This is not easy for a single microstrip antenna without sufficient diffraction in free space. References [56, 57] employed a $2 \times 2$ antenna array as the source to increase the radiating aperture so that the directivity is dramatically enhanced. The simulation model and prototype are shown in Figures 5(d) and 5(e), respectively. It should be noted that the increase of array antenna number does not lead to a large beam steering angle because the arrayed factor is only determined by the phase difference of antennas. Before effectively enhancing the rangeability of the transmission phase change greater than $360^{\circ}$, the beam steering angle is always a big problem of this scheme for SatCom use. We think that it should be more efficient to form a cavity for beam steering by using a GRIN total reflection surface rather than a GRIN partial reflection surface [58]. This unproved idea is left as an open issue here.

5.2. Leaky Wave Scheme. Both the reflectarray and superstrate schemes separate the antenna feed and beam steerer and thus the microwave diffraction region in between increases the overall antenna volume. An ideal way is to integrate beam radiation and shaping functions. Leaky wave (LW) scheme may be a good option in that a leaky mode in a transmission line may travel to some distance with a fixed propagation constant and gradually couple energy out. The distributed LW radiation produces a focused beam and 


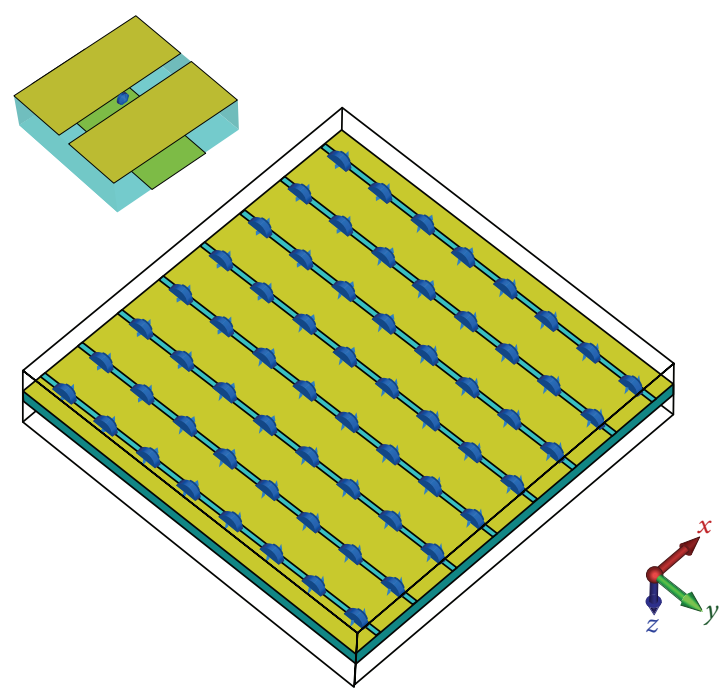

(a)

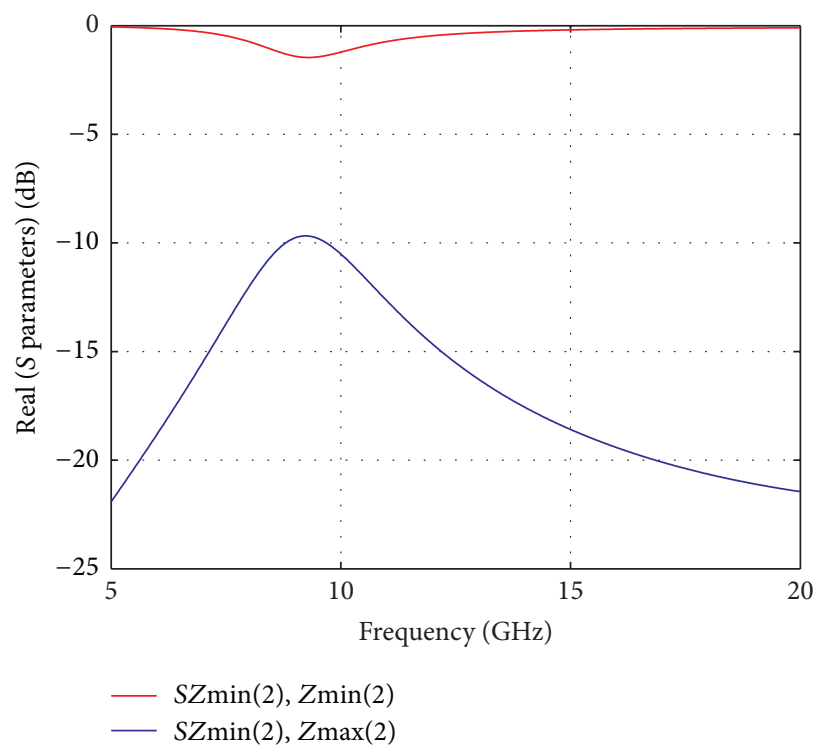

(b)

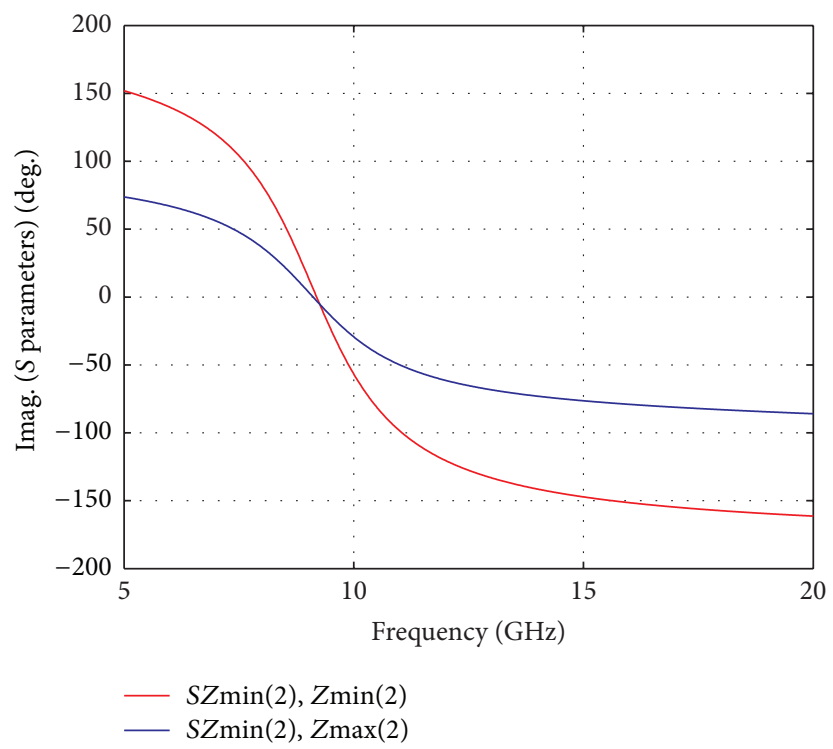

(c)

FIGURE 4: (a) A simulation model of tunable metamaterial superstrate with lumped varactor (in the inset is a unit cell); the transmission (b) amplitude and (c) phase versus frequency.

results in a highly directive gain. Recent activities in LW antenna have been stimulated by the surge of interest in MTMs, which has motivated new types of MTM structures as well as new ways to construct LW antenna. For radiation in both forward and backward direction, conventional LW antenna regularly operates with harmonics which requires complex narrow-band feeding circuits and fails to radiate a true broadside radiation pattern. To overcome this, the socalled composite right/left-handed (CRLH) transmission line (TL) was proposed to explore full-angle beam scanning.

In general, a lossless CRLH TL can be described by an equivalent circuit with four lumped parameters: series inductance $L_{R}$, shunt capacitance $C_{R}$, series capacitance $C_{L}$, and shunt inductance $L_{L}$ [59]. In a conventional TL, for example, the microstrip TL, the fundamental mode only has the first two elements in the equivalent circuit and corresponds to the right-handed $(\mathrm{RH})$ branch of the dispersion curve. The $\mathrm{RH}$ branch has positive phase velocity and corresponds to forward wave propagation. In contrast, the latter two elements are correspondent to the left-handed (LH) branch for backward wave propagation. The two branches of dispersion curves reach the series resonant frequency $\omega_{\text {se }}$ and shunt resonant frequency $\omega_{\text {sh }}$, respectively, while approaching the $\Gamma$ point for broadside radiation. By appropriately structuring a CRLH TL, it is possible to make them identical so that a smooth transition from the $\mathrm{LH}$ branch to the $\mathrm{RH}$ branch of 


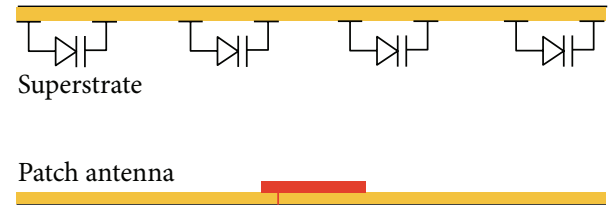

(a)

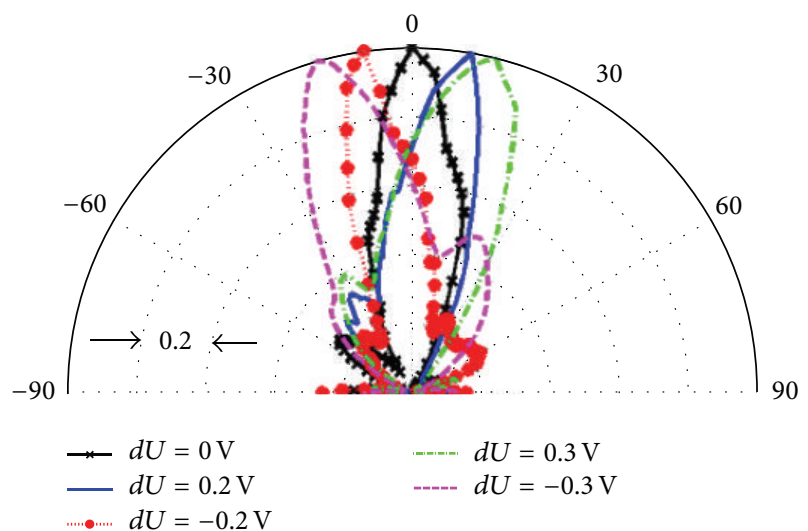

(c)

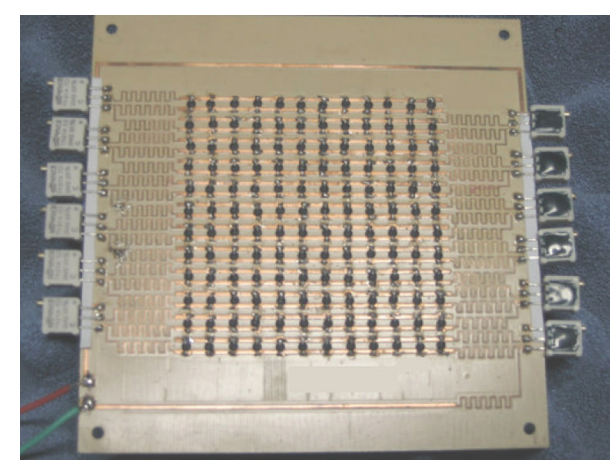

(b)

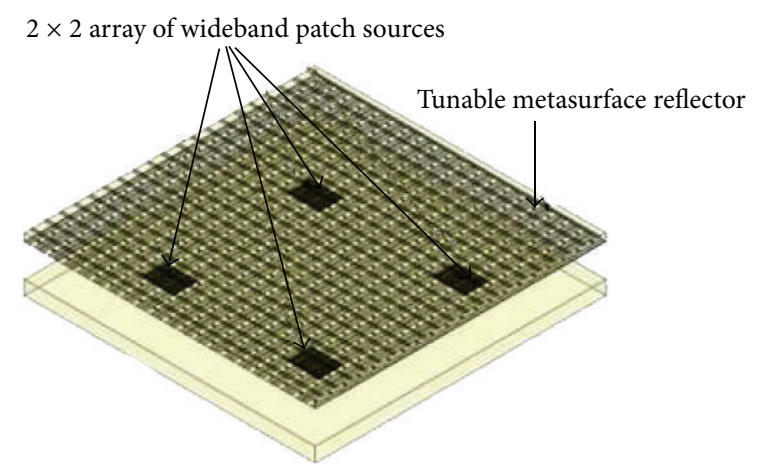

(d)

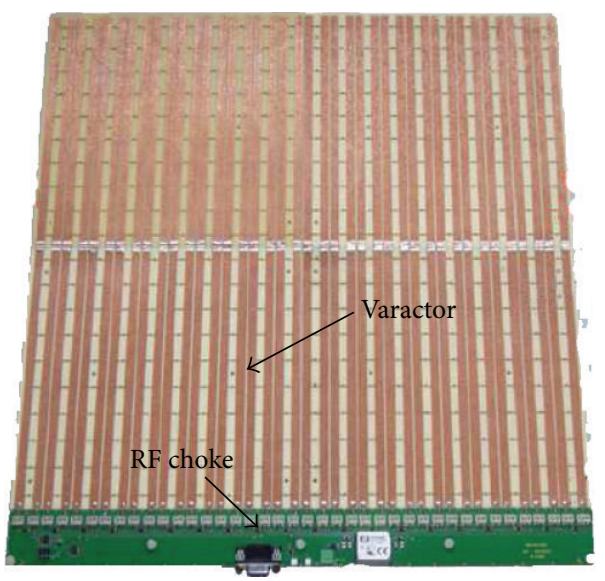

(e)

FIGURE 5: (a) Schematics of the cavity composed of a perfect-electric conductor (PEC) and an electronically phase varying partial reflection surface; (b) the prototype photo showing the capacitive grid side with biasing network; and (c) the measured gain patterns under different biasing condition [55] (reproduced by permission of the Institution of Engineering \& Technology). (d) A generalized cavity with $2 \times 2$ antenna array excitation and (e) the system equipped with biasing network $[56,57]$ (reproduced by permission of John Wiley and Sons).

the dispersion curve makes a perfect broadside radiation as well as the full-angle radiation [60].

Early demonstrations of beam steering with MTM LW antenna were mostly by frequency scanning, which has no mechanical device and low complexity of circuitry and wiring. Without any tunable element, the dispersion diagram that describes the relationship between frequency and propagation constant can be numerically simulated one time to predict the beam radiation angle. However, for bandwidth reason, beam scanning at a fixed frequency is certainly desired for SatCom. Figure 6 shows an important demonstration of an electronically steerable leaky wave antenna [61-63]. The antenna is based on the classical 2D CRLH transmission line, for example, mushroom structure. Varactor diodes incorporated inside allow electronic controlling of the reflection phase and the surface wave properties. Because 


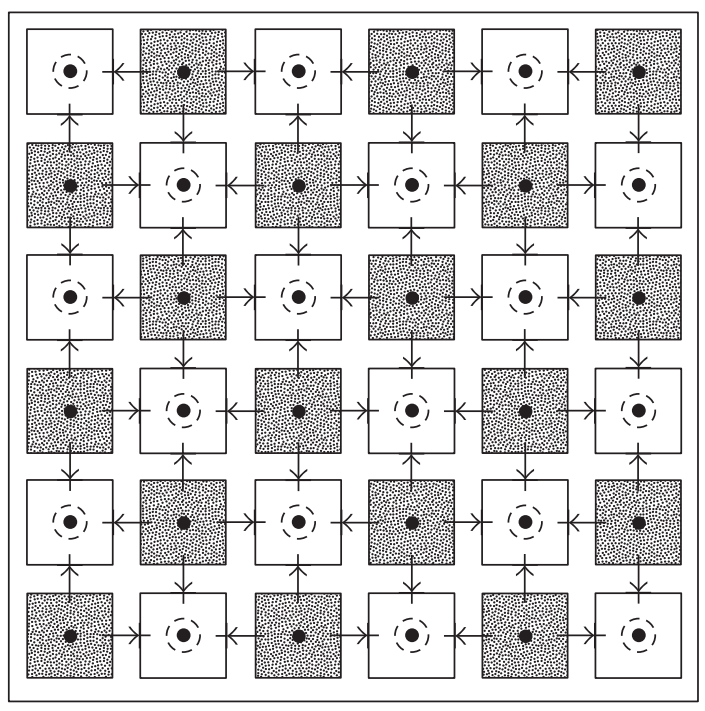

(a)

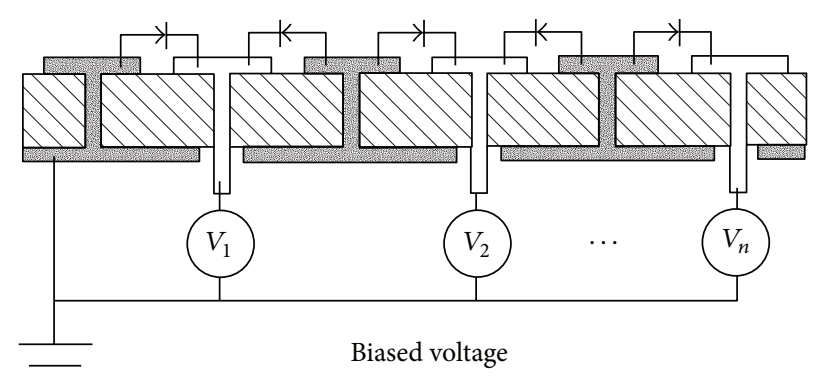

(b)

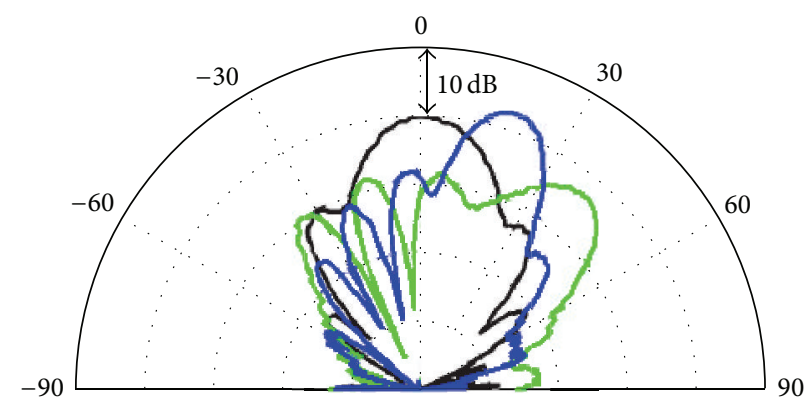

(c)

Figure 6: (a) The top view and (b) side view of an electrically tunable impedance surface with special arrangement of varactors and (c) the radiation patterns for three different phase gradients $[63,64]$ (reproduced by permission of the Institution of Engineering \& Technology).

the MTM cell is less than a quarter of wavelength, there is much great freedom to independently control the magnitude and phase of the leaky wave to optimize the effective aperture over the entire scanning range.

The way of arranging and feeding CRLH LW structures makes the radiation beam shape different. A 1D CRLH TL LW antenna makes a fan-shaped beam narrow in the scanning plane and fat in the transverse plane. Exciting a 2D CRLH TL LW antenna from the center makes a conical beam. A pencilshaped beam in the need of SatCom can be achieved by placing many $1 D$ CRLH LW structures parallelly in a row [65]. The narrow beam in the transverse plane is a result of phased array. It should be noted that 2D CRLH TL LW antenna shown in Figure 6 is azimuthally isotropic and effectively homogeneous. In theory, feeding a circularly shaped 2D CRLH TL from any azimuthal angle can achieve 1D fullangle beam steering within the corresponding propagation plane. In this way, an azimuthally switchable antenna can be achieved although this does not fully meet the requirement of continuous scanning for practical SatCom use.

5.3. Scatterer Scheme. The last, but probably the most impactful scheme of using MTMs, is the scattering scheme by which a new start-up Kymeta commercialized their solution to MOST [66]. The basic concept of scatterer scheme is to realize high gain beam steering by selectively switching on/off thousands of waveguide-fed tiny microresonators. The basic antenna architecture consists of a wave-propagating structure and many surface scattering elements in the proximity. The antenna reconfigurability is achieved by coupling and decoupling the scattering elements and the wave-propagating structure. A snap shot of the animation provided by [67] is shown in Figure 7. It can be seen that the surface wave propagating in the waveguide locally radiates out by activating the MTM cells to form a reconfigurable grating. The pattern of the activation determines the shape and direction of the radiated beam. Changing the pattern of activated elements changes the shape and direction of the beam.

There are many different terminologies in media or literatures to describe this technology, such as surface scattering antenna and holographic antenna. Actually the fundamental idea is very similar to the leaky wave antenna concept in the previous section. If there is any difference, in a narrow sense, a leaky wave antenna is inclined to be with a regular transmission line which guides leaky but stable Bloch modes with complex propagation constant. A half-width leaky 


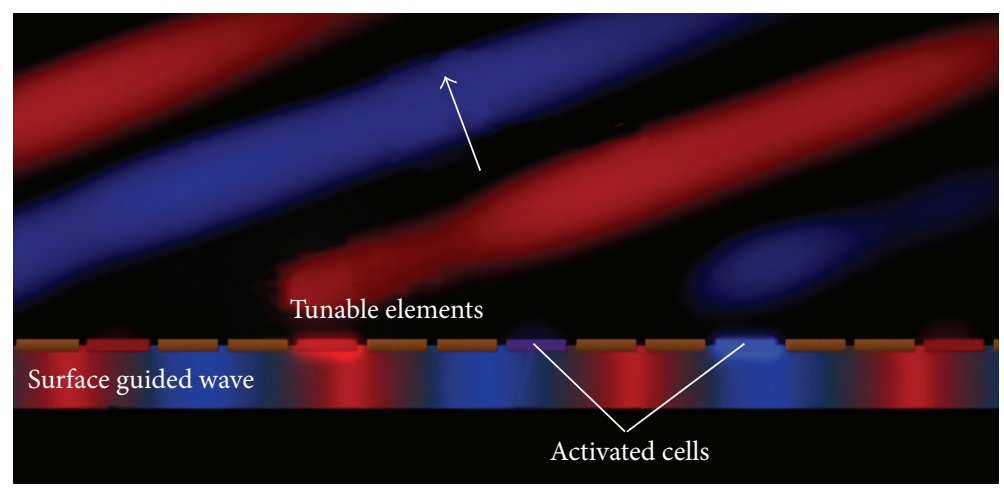

FIGURE 7: A snapshot of the wave radiating out from the microwave waveguide with selected cells activated [68] (used courtesy of Kymeta Corporation (C2015).

wave antenna connected with a set of computer-controlled switches is numerically simulated to realize configurability in a digital control manner [68].

It is the advanced digital circuit that permits thousands of switches to be arbitrarily switched on/off. However, a large number of switches mean that the possible combinations easily amount to an astronomical figure. Moreover, the mutual coupling between adjacent MTM cells makes the radiation problem more complicated. An efficient algorithm is badly needed to know the activation pattern for various beam steering angles before putting the antenna into use. Johnson et al. derived a discrete-dipole approximation (DDA) model to quickly predict the radiation of a 2D MTMs at a much reduced computational cost to full-wave simulation and at much greater fidelity than those precedent models [69]. Aside from beam steering angle, the sidelobe is also an important criterion to optimize the radiation pattern. An optimization algorithm for the far field pattern has been developed to suppress sidelobe in software without any onadaptive hardware modification [70].

\section{Trends and Challenges}

Exploring new schemes and conducting system-level optimization are undoubtfully the future trends in common. In this paper, we specially highlighted the "metamaterials" approaches not because of its popularity in academic society but its potential in the next generation of SatCom antenna. It can be easily understood by recalling the procedure of beam shaping. Given a complete description of the desired pattern, the excitation distribution of a continuous or a discrete source antenna system can be determined by Fourier transform method [71]. According to sampling theorem, the antenna (discrete microwave source) spacing and tunability determine the quality of the reconstruction of the far field pattern, or say how closely the reconstructed far field pattern approximates to the desired pattern. Ideally the spacing and size of the elemental radiators are sufficiently small and both their amplitude and phase are arbitrarily tunable. This is a general design guideline no matter how to technically engineer the near field pattern, using dish, reflector, superstrate, and whatever. MTMs set a direction flag to acquire finer and exquisite tunability in the near field. The future SatCom antenna may seek the largest freedom of tunability under the constraint of the current fabrication technologies and the processing capability of peripheral controlling circuit. Another straightforward guideline is to simplify the system as much as possible. The fewer the component, the RF signal passes through, the less the microwave attenuation, the power consumption, and the heat production. In the following text, we briefly talk about a few trends and challenges from the aspect of engineering.

6.1. Customization. We reviewed various implementation schemes of MOST antenna with common considerations. However, the service diversity of MOST actually needs to customize the antenna designs to suit mechanical requirements associated with construction strength and easy installation. In this way, different specifications are not of the same importance so that compromise can be made to take care of the critical ones. Land vehicles call for low profile and lightweight antenna equipment to share the limited space with many other functional antennas and maintain the beauty of framework. On shipborne terminals, the space for antenna installation is comfortable, but all ship antennas suffer stress from vibration and sloping by strong wins and corrosion from sea salt [72]. Protective measures should be taken. Besides, in some cases (e.g., a large ocean-going ship) in which only transmitting or receiving function is required, very simple low-gain omnidirectional antennas operating at $L$-band or $C$-band are also acceptable at the expense of bandwidth [73]. On trains communicating with geostationary satellite solution, the tracking of the satellite on the move requires different antenna elevation angles which are dependent on the location of the antenna relative to the equator above which the satellite is located. Antenna must have a wide elevation angle range to ensure maximal flexibility in choosing the satellite of operation [74]. The selection, customization, and system-level simulation of SatCom antenna will be more and more sophisticated as the mechanics, platforms, functions, and services become diverse. 
6.2. Capacity Enhancement. SatCom service provider is looking forward to the bandwidth enhancement through raising the operating frequency at $K_{a}$ band or beyond $K_{a}$ (e.g., V band) $[75,76]$. Unfortunately, the severe signal fading above $10 \mathrm{GHz}$ due to the strong attenuation through atmosphere deteriorates the quality of service and hardly satisfies the availability recommended by ITU. Hence, fade mitigation technique (FMT) is vital to make these operation frequency feasible for MOST. Reference [77] outlined a variety of FMTs for SatCom systems operating in the $10 \mathrm{GHz}$ to $50 \mathrm{GHz}$ spectral region. For example, among them, the frequency diversity technique uses high frequency normally and switches over to spare channels at lower frequency band when deep fades occur. For such switching capability, the reconfigurability deserves special attention for antennas on the ground mobile terminals.

The multiple-input multiple-output (MIMO) technology is another attractive approach to enhancing SatCom capacity. Because of the promise of very high data rates at no cost of extra spectrum and transmit power, the success of MIMO technology in terrestrial communications stimulated its application in SatCom to benefit the spatial multiplexing and diversity $[78,79]$. The multiple elemental antenna must be compact enough to fit user equipment, supportive to multiband operation, and well isolated from mutual interference [80]. Also, the large ground antenna separation in the need of the long-distance SatCom links is also expected to be minimized [81].

6.3. Reconfigurability. The antenna reconfigurability is valuable because it means that, after leaving the factory, the operation frequency and/or radiation properties can still be dynamically modified in a controllable and reversible manner to respond to the operating requirements. Although many physical mechanisms had been proposed to enable this, only a few technologies are relatively matured in industry, such as varactors, PIN diode switches, RF MEMS switches, and liquid crystals. The pros and cons had been extensively discussed in previous publications $[39,82,83]$. For brevity, we only highlight those critical aspects related to the SatCom applications according to our limited experience. The selection of tunable elements has to consider the following factors: analog or digital control, multiple-end or single-ended biasing, operation voltage, linearity, component size, self-resonating frequency, voltage or current constraint, power consumption, and readiness of fabrication technology. In general, it is not easy to implement analog signals if hundreds of inputs are required. Digital control is substantially flexible. Varactor mainly features in low power consumption and high speed operation because the $\mathrm{PN}$ junction inside is reversely biased and carrier depletion effect is used. The PIN diode is suitable to dynamically open/short a circuit with a moderate biasing voltage (typically $<10 \mathrm{~V}$ ). But the performance becomes poor as frequency increases because of the limitation of carrier lifetime. The MEMS switch can achieve very high isolation for ideal on/off switching but the action of switching is slow and the biasing voltage is usually very high (typically $>20 \mathrm{~V}$ ). Liquid crystal is attractive because of its simple biasing network. But high voltage biasing and special filling process are needed [84]. All these enabling technologies are still experiencing development to remedy their own defect.

6.4. Dense Integration. Highly monolithic microwave integrated circuits (MMIC) integration definitely represents a future trend to construct SatCom antenna. The integration of microwave components into a single chip outperforms an assembled system in time, cost, efficiency, reliability, complexity, and, more importantly, compactness [28, 30, 85]. The merits of MMIC have been identified on GaAs platform by nearly 30 years [86]. But one thing that changes the world is the advancement of Si-based technologies [33, 87]. The conventional constraint of using Si technologies in speed had been solved by transistor size reduction. The advantage of using Si becomes prominent due to the low fabrication cost and matured fabrication technology. It should be noted that the mainstream Si wafer size is as large as 8 or 12 inch, which is comparable to the aperture of a SatCom antenna at or above $K_{a}$ band. It is an ambitious but possible idea to use a full Si wafer for SatCom purpose in the future. Finally, microwave photonics had experienced rapid growth on $\mathrm{Si}$ platform. The merging of microwave and photonics offers a large possibility to further push SatCom antenna technology to smaller dimension and more novel functions. Some interesting proposal and demonstrations are in progress [88, 89].

\section{Conclusion}

Because of the particularities, satellite communication has special needs in antenna size/weight, gain, sidelobe level, mobility, and so forth. Within a limited radiation aperture, high gain should be maintained in all possible linking directions. In addition, sidelobe suppression should be implemented to avoid signal leakage or undesired crosstalk. Fast tracking speed must be achieved to set up the link and conquer any accidental disturbance during communication. All these requirements call for careful consideration in the planning stage including the fundamental concept. This paper reviewed the major methodologies in literatures to achieve dynamic beam scanning for the mobile satellite communications purpose and attempted to understand the obstacles or the last mile of each toward realistic application.

\section{Conflict of Interests}

The authors declare that there is no conflict of interests regarding the publication of this paper.

\section{Acknowledgments}

The authors thank staffs of Aerospace project office, Agency for Science Technology and Research (A STAR), for their continuous support, and the members in the lead sponsor, Defence Science \& Technology Agency (DSTA), for their enlightening discussion. The authors also thank all the researchers in the reference list; without their materials and insights the authors are not able to compose this paper and put forward their views. This work is supported by 
the Cycle-7 consortium projects (Grant no. 112155 0715) funded by A ${ }^{*}$ STAR aerospace programme.

\section{References}

[1] http://www.sia.org/wp-content/uploads/2014/09/SSIR-September-2014-Update.pdf.

[2] Satllite Evolution Group, A New Era for the MSS Market, Satllite Evolution Group, 2014.

[3] B. R. Elbert, The Satellite Communication Applications Handbook, Artech House, Boston, Mass, USA, 2nd edition, 2004.

[4] B. R. Elbert, Introduction to Satellite Communication, Artech House, 3rd edition, 2008.

[5] V. Rabinovich, N. Alexandrov, and B. Alkhateeb, Automotive Antenna Design and Applications, CRC Press, Boca Raton, Fla, USA, 2010.

[6] S. Ohmori, "Vehicle antennas for mobile satellite communications," IEICE Transactions, vol. E74, pp. 3210-3221, 1991.

[7] R. E. Sheriff and Y. F. Hu, Mobile Satellite Communication Networks, John Wiley \& Sons, Ltd, New York, NY, USA, 2001.

[8] D. Minoli, Innovations in Satellite Communications and Satellite Technology, the Industry Implications of DVB-S2X, High throughput Satellites, Ultra HD, M2M, and IP, John Wiley \& Sons, Hoboken, NJ, USA, 2015.

[9] V. Weerackody and E. G. Cuevas, "Technical challenges and performance of satellite communications on-the-move systems," Johns Hopkins APL Technical Digest, vol. 30, no. 2, pp. 113-121, 2011.

[10] A. Imbriale, S. Gao, and L. Boccia, Space Antenna Handbook, Wiley, Hoboken, NJ, USA, 2012.

[11] B. Basari, K. Saito, M. Takahashi, and K. Ito, "Antenna system for land mobile satellite communications," in Satellite Communication, N. Diodato, Ed., chapter 2, InTech, 2010.

[12] ITU, "Attenuation by atmospheric gases $\mathrm{P}$ series, radiowave propagation," Recommendation ITU-R P.676-9, 2012.

[13] L. Marcellini, R. Lo Forti, and G. Bellaveglia, "Future developments trend for $\mathrm{Ku}$ and $\mathrm{Ka}$ antenna for satcom on the move," in Proceedings of the 5th European Conference on Antennas and Propagation (EUCAP '11), pp. 2346-2350, Rome, Italy, April 2011.

[14] J. Brand, "Practical on-the-move satellite communications for present and future mobile warfighters," in Proceedings of the Military Communications Conference (MILCOM '05), pp. 625629, Atlantic City, NJ, USA, October 2005.

[15] M. R. Chartrand, Satellite Communications for the Nonspecialist, SPIE Press, Washington, DC, USA, 2004.

[16] M. W. Bai, K. A. Kingston, H. R. Malone, J. G. Doggett, R. P. Vidano, and E. R. YIngling, "Method and apparatus for eleminating keyhole problemof an azimuth-elevation gimbal antenna," US Patent no.: US6285338B1, 2001.

[17] A. C. Densmore, V. Jamnejad, and K. E. Woo, "Satellite-tracking millimeter wave reflector antenna system for mobile satellite tracking," US Patent no.: US RE37218E, 2001.

[18] D. J. Legare and D. M. Hummel, "Antenna for compact satellite terminal," US Patent no.: US7859479B2, 2010.

[19] L. King and J. Boardson, "Enclosed antenna system for receiving broadcasts from multiple sources," US Patent no. US8368611B2, 2010.

[20] Y.-B. Jung, S.-Y. Eom, and S.-I. Jeon, "Novel antenna system design for satellite mobile multimedia service," IEEE Transactions on Vehicular Technology, vol. 59, no. 9, pp. 4237-4247, 2010.
[21] http://www.most-sys.com/.

[22] Y.-B. Jung, A. V. Shishlov, and S.-O. Park, "Cassegrain antenna with hybrid beam steering scheme for mobile satellite communications," IEEE Transactions on Antennas and Propagation, vol. 57, no. 5, pp. 1367-1372, 2009.

[23] N. C. Karmakar and M. E. Bialkowski, "Electronically steerable array antennas for mobile satellite communications-a review," in Proceedings of the IEEE International Conference on Phased Array Systems and Technology, pp. 81-84, Dana Point, Calif, USA, May 2000.

[24] D. Ehyaie, Novel approaches to the design of phased array antennas [Ph.D. thesis], University of Michigan, Ann Arbor, Mich, USA, 2011.

[25] D. P. Spano and D. A. Green, "Low profile antenna positioner for adjusting elevation and azimuth," US Patent no. US6204823B1, 2011.

[26] G. D. Han, B. Du, W. Wu, and B. Yang, "A novel hybrid phased array antenna for satellite communication on-the-move in $\mathrm{Ku}$ band," IEEE Transactions on Antennas and Propagation, vol. 63, no. 4, pp. 1375-1383, 2015.

[27] R. Baggen, S. Holzwarth, M. Bottcher, and M. Eube, "Phased array technology: trends \& developments," in Proceedings of the German Microwave Conference (GeMiC '05), Ulm, Germany, April 2005.

[28] L. C. Godara, "Applications of antenna arrays to mobile communications. Part I. Performance improvement, feasibility, and system considerations," Proceedings of the IEEE, vol. 85, no. 7, pp. 1031-1060, 1997.

[29] S. Ohmori, "Phased array antennas for mobile communications," Annals of Telecommunications, vol. 54, no. 1, pp. 93-102, 1999.

[30] I. Kaplan, I. Marinov, A. Gal et al., "Electronically beam steerable antennas for broadband satellite communications," in Proceedings of the 8th European Conference on Antennas and Propagation (EuCAP '14), pp. 2450-2454, The Hague, The Netherlands, April 2014.

[31] S. Vaccaro, D. L. del Rio, J. Padilla, and R. Baggen, "Low cost $\mathrm{Ku}$-band electronic steerable array antenna for mobile satellite communications," in Proceedings of the 5th European Conference on Antennas and Propagation, pp. 2362-2366, Rome, Italy, April 2011.

[32] L. Josefsson and P. Persson, Conformal Array Antenna Theory and Design, IEEE Press, Wiley-Interscience, 2006.

[33] H. Schippers, J. Verpoorte, P. Jorna et al., "Broadband conformal phased array with optical beam forming for airborne satellite communication," in Proceedings of the IEEE Aerospace Conference (AC '08), pp. 1-17, IEEE, Big Sky, Mont, USA, March 2008.

[34] F. Amoozegar, V. Jammnejad, T. Pham, and R. Cesarone, "Trends in development of broadband phased arrays for space applications," IEEEAC Paper 1335, 2003.

[35] P. Chen, W. Hong, H. Zhang, J. X. Chen, H. J. Tang, and Z. Chen, "Virtual phase shifter array and its application on ku band mobile satellite reception," IEEE Transactions on Antennas and Propagation, vol. 63, no. 4, pp. 1408-1416, 2015.

[36] Y. Rahmat-Samii and A. Densmore, "A history of reflector antenna development: past, present and future," in Proceedings of the SBMO/IEEE MTT-S International Microwave and Optoelectronics Conference (IMOC '09), pp. 17-23, Belem, Brazil, November 2009.

[37] J. Shaker, M. R. Chaharmir, and J. Ethier, Reflectarray Antennas: Analysis, Design, Fabrication and Measurement, Artech House Antennas and Propagation Library, Artech House, 2013. 
[38] V. Ziegler, "Public report on project results of the project RETINA," Aeronautics \& Space, 2008, http://www.transport-research.info/Upload/Documents/201003/20100311_104621_85168_ Retina_Deliverable_D7-5[1].pdf, http://www.transport-research info/web/projects/project_details.cfm?id=11258.

[39] S. V. Hum and J. P. Carrier, "Reconfigurable reflectarrays and array lenses for dynamic antenna beam control: a review," IEEE Transactions on Antennas and Propagation, vol. 62, no. 1, pp. 183-198, 2013.

[40] M. E. Bialkowski and K. H. Sayidmarie, "Investigations into phase characteristics of a single-layer reflectarray employing patch or ring elements of variable size," IEEE Transactions on Antennas and Propagation, vol. 56, no. 11, pp. 3366-3372, 2008.

[41] B. O. Zhu, J. M. Zhao, and Y. J. Feng, "Active impedance metasurface with full $360^{\circ}$ reflection phase tuning," Scientific Reports, vol. 3, article 3059, 2013.

[42] M. E. Bialkowski and J. A. Encinar, "Reflectarrays: potentials and challenges," in Proceedings of the International Conference on Electromagnetics in Advanced Applications (ICEAA '07), pp. 1050-1053, Torino, Italy, September 2007.

[43] M. Y. Ismail and M. I. Abbasi, "Analysis of design optimization of bandwidth and loss performance of reflectarray antennas based on material properties," Modern Applied Science, vol. 4, no. 1, 2010.

[44] V. G. Veselago, "Electrodynamics of substances with simultaneously negative values of $\epsilon$ and $\mu$," Soviet Physics Uspekhi, vol. 10, no. 4, pp. 509-514, 1968.

[45] J. B. Pendry, D. Schurig, and D. R. Smith, "Controlling electromagnetic fields," Science, vol. 312, no. 5781, pp. 1780-1782, 2006.

[46] P.-H. Tichit, S. N. Burokur, and A. D. Lustrac, "Antenna design concepts based on transformation electromagnetics approach," Radioengineering, vol. 21, no. 4, pp. 954-962, 2012.

[47] N. Kundtz and D. R. Smith, "Extreme-angle broadband metamaterial lens," Nature Materials, vol. 9, no. 2, pp. 129-132, 2010.

[48] Q. Cheng, T. J. Cui, W. X. Jiang, and B. G. Cai, "An electromagnetic blackhole made of metamaterials," http://arxiv.org/abs/ 0910.2159v2.

[49] D. R. Smith, J. J. Mock, A. F. Starr, and D. Schurig, "Gradient index metamaterials," Physical Review E, vol. 71, no. 3, Article ID 036609, 2005.

[50] A. Ourir, S. N. Burokur, and A. De Lustrac, "Phase-varying metamaterial for compact steerable directive antennas," Electronics Letters, vol. 43, no. 9, pp. 493-494, 2007.

[51] D. Y. Li, Z. Szabo, X. M. Qing, E.-P. Li, and Z. N. Chen, "A high gain antenna with an optimized metamaterial inspired superstrate," IEEE Transactions on Antennas and Propagation, vol. 60, no. 12, pp. 6018-6023, 2012.

[52] H. Chen, B.-I. Wu, L. Ran, T. M. Grzegorczyk, and J. A. Kong, "Controllable left-handed metamaterial and its application to a steerable antenna," Applied Physics Letters, vol. 89, no. 5, Article ID 053509, 2006.

[53] H. F. Ma, G. Z. Wang, W. X. Jiang, and T. J. Cui, "Independent control of differently-polarized waves using anisotropic gradient-index metamaterials," Scientific Reports, vol. 4, article 6337, 2014.

[54] H. F. Ma, X. Chen, X. M. Yang, W. X. Jiang, and T. J. Cui, "Design of multibeam scanning antennas with high gains and low sidelobes using gradient-index metamaterials," Journal of Applied Physics, vol. 107, no. 1, Article ID 014902, 2010.

[55] A. Ourir, S. N. Burokur, and A. De Lustrac, "Electronic beam steering of an active metamaterial-based directive subwavelength cavity," in Proceedings of the 2nd European Conference on
Antennas and Propagation (EuCAP '07), p. 257, Edinburgh, UK, November 2007.

[56] S. N. Burokur, J.-P. Daniel, P. Ratajczak, and A. de Lustrac, "Lowprofile frequency agile directive antenna based on an active metasurface," Microwave and Optical Technology Letters, vol. 53, no. 10, pp. 2291-2295, 2011.

[57] S. N. Burokur, A. Ourir, A. de Lustrac, and R. Yahiaoui, "Metasurfaces for high directivity antenna applications," in Metamaterial, X. Y. Jiang, Ed., chapter 20, InTech, Rijeka, Croatia, 2012.

[58] R. Guzmán-Quirós, J. L. Gómez-Tornero, A. R. Weily, and Y. J. Guo, "Electronically steerable 1-d fabry-perot leaky-wave antenna employing a tunable high impedance surface," IEEE Transactions on Antennas and Propagation, vol. 60, no. 11, pp. 5046-5055, 2012.

[59] C. Caloz and T. Itoh, Electromagnetic Metamaterials: Transmission Line Theory and Microwave Applications, WileyInterscience, John Wiley \& Sons, 2006.

[60] K. Sakoda and H. Zhou, "Role of structural electromagnetic resonances in a steerable left-handed antenna," Optics Express, vol. 18, no. 26, pp. 27371-27386, 2010.

[61] D. F. Sievenpiper, "Forward and backward leaky wave radiation with large effective aperture from an electronically tunable textured surface," IEEE Transactions on Antennas and Propagation, vol. 53, no. 1, pp. 236-247, 2005.

[62] D. F. Sievenpiper, J. H. Schaffner, H. J. Song, R. Y. Loo, and G. Tangonan, "Two-dimensional beam steering using an electrically tunable impedance surface," IEEE Transactions on Antennas and Propagation, vol. 51, no. 10, pp. 2713-2722, 2003.

[63] D. Sievenpiper and J. Schaffner, "Beam steering microwave reflector based on electrically tunable impedance surface," Electronics Letters, vol. 38, no. 21, pp. 1237-1238, 2002.

[64] D. Sievenpiper, "Meta element antenna and array," US Patent no. US 20040227667A1, 2004.

[65] H. V. Nguyen, S. Abielmona, A. Rennings, and C. Caloz, "Pencil-beam full-space scanning 2D CRLH leaky-wave antenna array," in Proceedings of the International Symposium on Signals, Systems and Electronics (ISSSE '07), pp. 139-142, IEEE, Québec, Canada, August 2007.

[66] A. Bily, A. K. Boardman, B. J. Hang et al., "Surface scattering antennas," US Patent no. US2012/0194399 A1, 2012.

[67] R. O. Ouedraogo, E. J. Rothwell, and B. J. Greetis, "A reconfigurable microstrip leaky-wave antenna with a broadly steerable beam," IEEE Transactions on Antennas and Propagation, vol. 59, no. 8, pp. 3080-3083, 2011.

[68] http://www.kymetacorp.com/technology/.

[69] M. C. Johnson, S. L. Brunton, N. B. Kundtz, and J. N. Kutz, "Sidelobe canceling for reconfigurable holographic metamaterial antenna," IEEE Transactions on Antennas and Propagation, vol. 63, no. 4, part 2, pp. 1881-1886, 2015.

[70] M. Johnson, P. Bowen, N. Kundtz, and A. Bily, "Discretedipole approximation model for control and optimization of a holographic metamaterial antenna," Applied Optics, vol. 53, no. 25, pp. 5791-5799, 2014.

[71] C. A. Balanis, Antenna Synthesis and Continuous Sources, John Wiley \& Sons, Hoboken, NJ, USA, 2005.

[72] S. D. Ilcev, "Antenna systems for mobile satellite applications," in Global Mobile Satellite Communications: For Maritime, Land and Aeronautical Applications, chapter 4, pp. 175-234, Springer, Berlin, Germany, 2005.

[73] S. D. Ilcev, "Low and medium-gain antennas for mobile satellite communications (MSC) and navigation," in Proceedings of the 
10th International Conference on Telecommunication in Modern Satellite Cable and Broadcasting Services (TELSIKS '11), vol. 2, pp. 697-700, IEEE, Niš, Serbia, October 2011.

[74] D. Oren, "Bringing broadband connectivity to trains with satellite communication," White Paper, Gilat Satellite Networks, 2015.

[75] K. Roebuck, Ka Band Satellite Communications: High-Impact Technology-What You Need to Know: Definitions, Adoptions, Impact, Benefits, Maturity, Vendors, Emereo Publishing Pty, 2011.

[76] E. K. Pfeiffer, T. Ernst, and A. Ihle, "Highly stable lightweight antennas for $\mathrm{Ka} / \mathrm{Q} / \mathrm{V}$-band and other advanced telecom structure concepts," in Proceedings of the 3rd European Conference on Antennas and Propagation (EuCAP '09), pp. 745-749, IEEE, Berlin, Germany, March 2009.

[77] A. D. Panagopoulos, P. D. M. Arapoglou, and P. G. Cottis, "Satellite communications at KU, KA, and V bands: propagation impairments and mitigation techniques," IEEE Communications Surveys \& Tutorials, vol. 6, no. 3, pp. 2-14, 2004.

[78] P.-D. Arapoglou, K. Liolis, M. Bertinelli, A. Panagopoulos, P. Cottis, and R. De Gaudenzi, "MIMO over satellite: a review," IEEE Communications Surveys \& Tutorials, vol. 13, no. 1, pp. 2751, 2011.

[79] T. D. Cola, D. Tarchi, and A. Vanelli-Coralli, "Future trends in broadband satellite communications: information centric networks and enabling technologies," International Journal of Satellite Communications and Networking, 2015.

[80] A. Jamil, M. Z. Yusoff, and N. Yahya, "Current issues and challenges of MIMO antenna designs," in Proceedings of the International Conference on Intelligent and Advanced Systems (ICIAS '10), pp. 1-5, IEEE, Kuala Lumpur, Malaysia, June 2010.

[81] Y. Rahmat-Samii and A. C. Densmore, "Technology trends and challenges of antennas for satellite communication systems," IEEE Transactions on Antennas and Propagation, vol. 63, no. 4, pp. 1191-1204, 2015.

[82] A. Petosa, "An overview of tuning techniques for frequencyagile antennas," IEEE Antennas and Propagation Magazine, vol. 54, no. 5, pp. 271-296, 2012.

[83] J. P. Turpin, J. A. Bossard, K. L. Morgan, D. H. Werner, and P. L. Werner, "Reconfigurable and tunable metamaterials: a review of the theory and applications," International Journal of Antennas and Propagation, vol. 2014, Article ID 429837, 18 pages, 2014.

[84] A. Choubey, F. Andros, and B. G. Sammakia, "Study of assembly processes for liquid crystal on silicon (LCoS) microdisplays," IEEE Transactions on Components and Packaging Technologies, vol. 28, no. 2, pp. 303-310, 2005.

[85] C.-H. Lee and J. Laskar, Compact Ku-Band Transmitter Design for Satellite Communication Applications: From System Analysis to Hardware Implementation, Springer, 2002.

[86] D. J. Connolly, K. B. Bhasin, and R. R. Romanofsky, "Monolithic microwave integrated circuit (MMIC) technology for space communications applications," NASA Technical Memorandum, 1987.

[87] M. A. Y. Abdalla, Metamaterials inspired CMOS tunable microwave integrated circuits for steerable antenna array [Ph.D. thesis], University of Toronto, Toronto, Canada, 2009.

[88] J. Verpoorte, H. Schippers, P. Jorna et al., "Architectures for ku band broadband airborne satellite communication antennas," in Proceedings of the 32nd ESA Antenna Workshop on Antennas for Space Applications, Noordwijk, The Netherlands, October 2010.
[89] M. Sotom, B. Benazet, A. Le Kernec, and M. Maignan, "Microwave photonic technologies for flexible satellite telecom payloads," in Proceedings of the European Conference on Optical Communication (ECOC '09), pp. 20-24, Vienna, Austria, September 2009. 

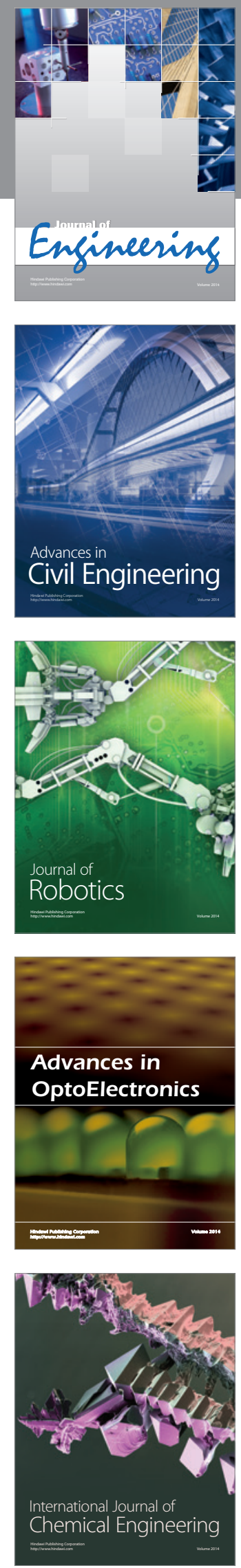

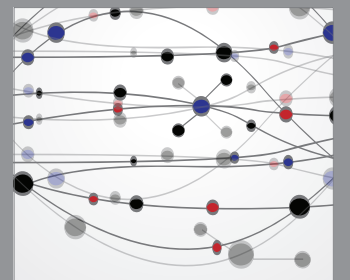

The Scientific World Journal
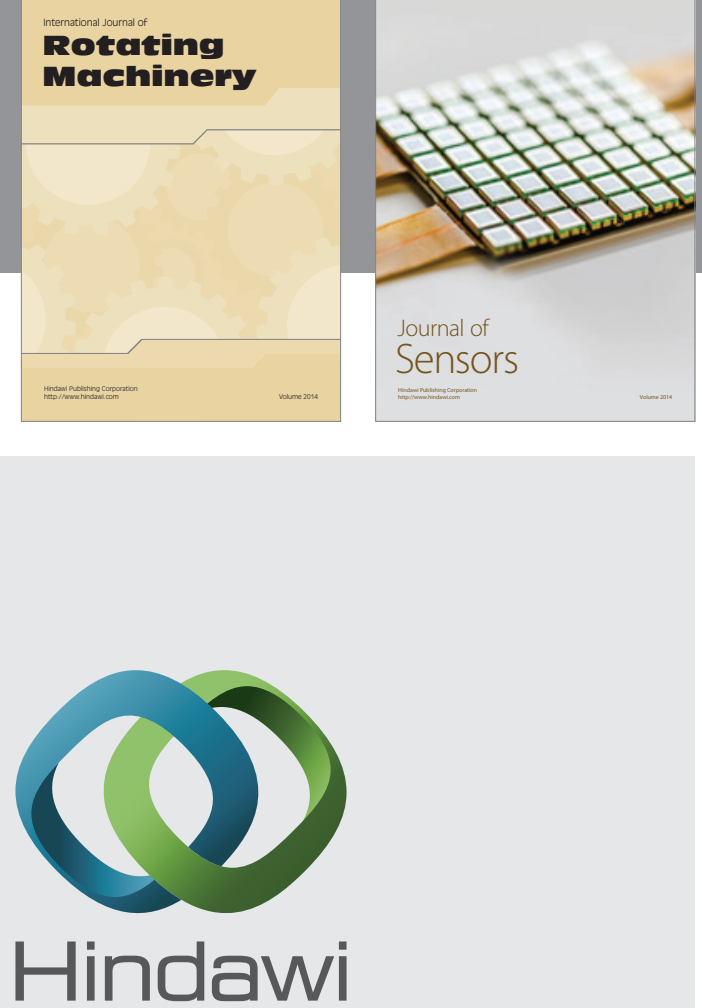

Submit your manuscripts at http://www.hindawi.com
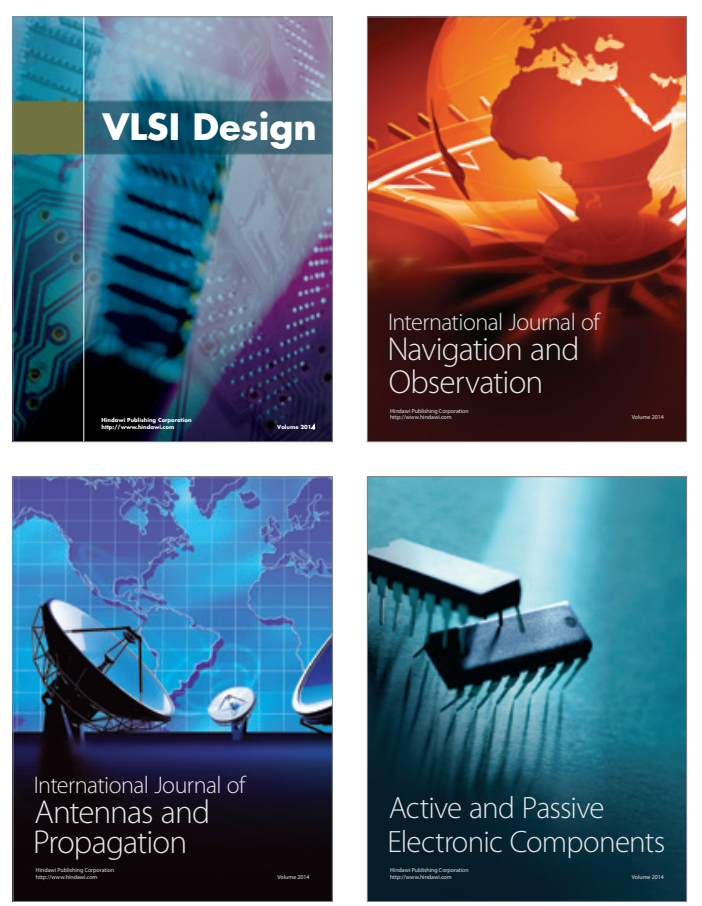
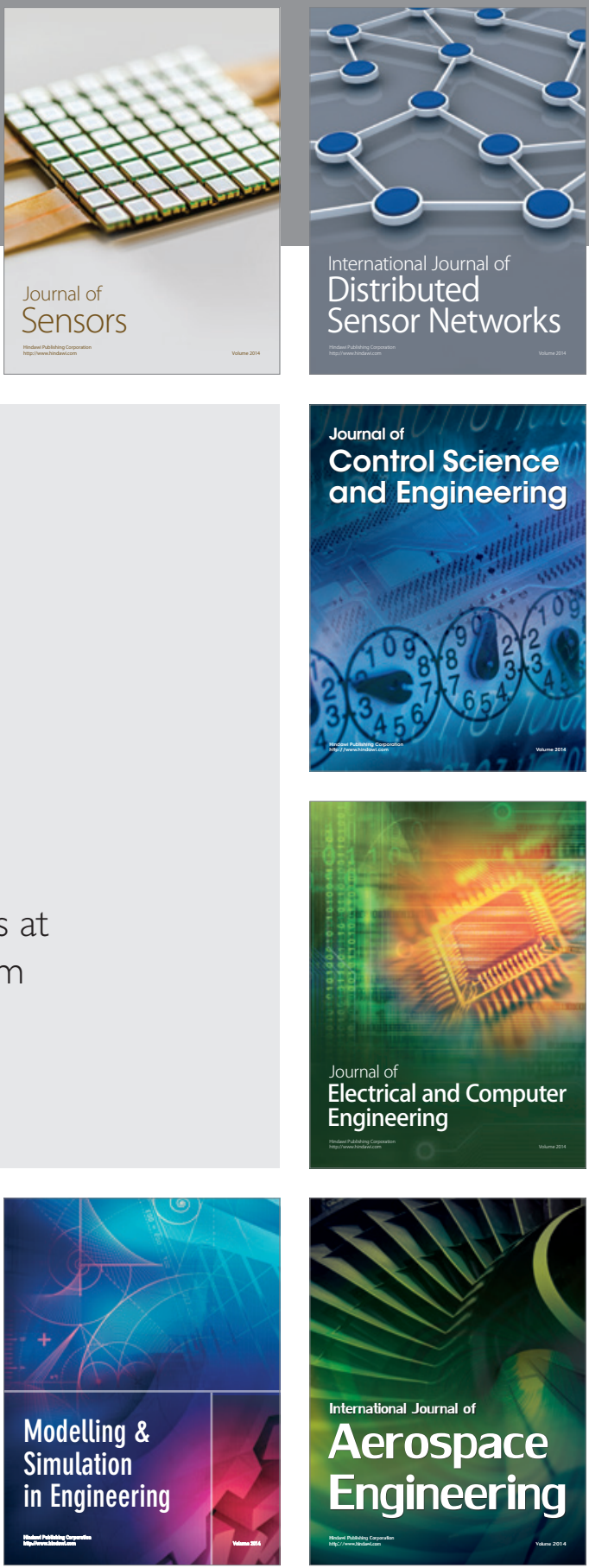

Journal of

Control Science

and Engineering
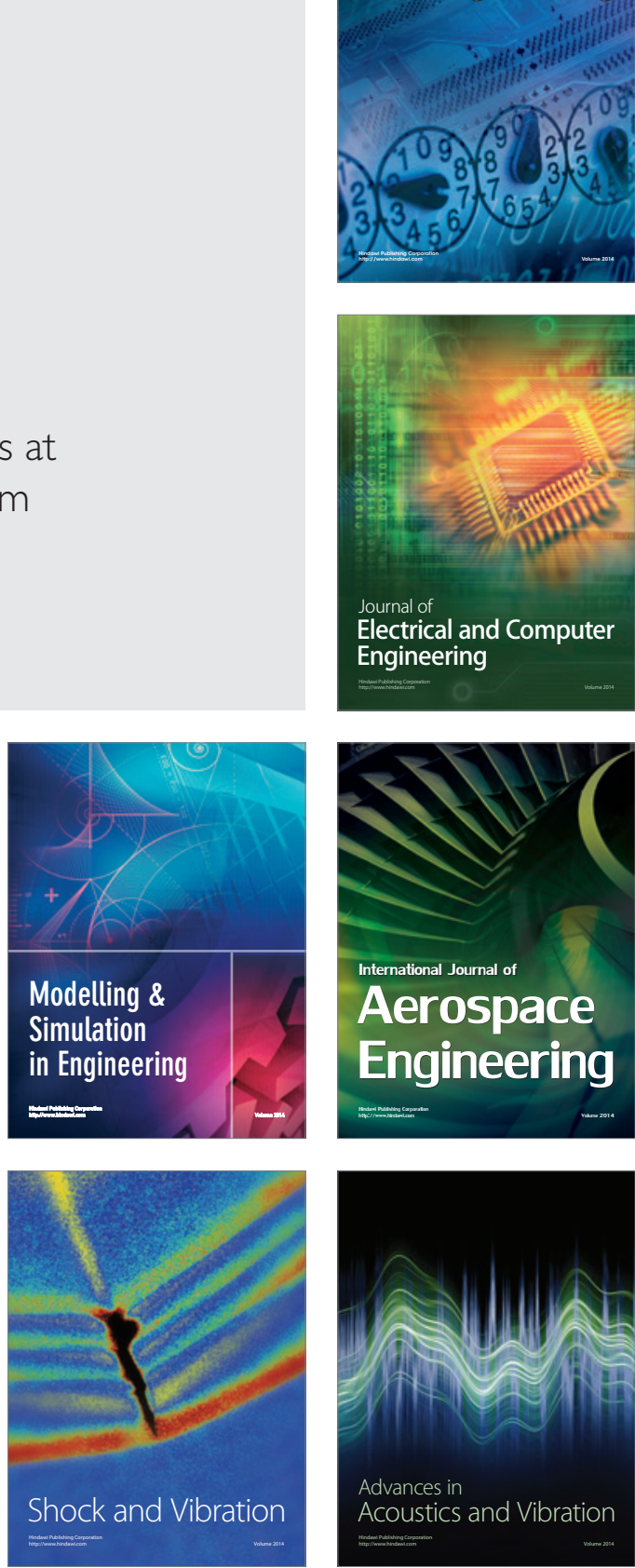